\title{
Multimodal imaging of aortic annulus and root geometry after valve sparing root reconstruction
}

\author{
Matthew Valdis ${ }^{1}$, Andrew Thain ${ }^{2}$, Philip M. Jones ${ }^{3,4}$, Ian Chan ${ }^{5}$, Michael W. A. Chu ${ }^{1}$ \\ ${ }^{1}$ Division of Cardiac Surgery, Department of Surgery, ${ }^{2}$ Division of Cardiology, Department of Medicine, ${ }^{3}$ Department of Anesthesia \& Perioperative \\ Medicine, ${ }^{4}$ Department of Epidemiology \& Biostatistics, ${ }^{5}$ Department of Radiology, Western University, London Health Sciences Centre, London, \\ Ontario, Canada \\ Correspondence to: Dr. Michael W. A. Chu, MD, FRCSC. Associate Professor of Surgery, B6-106 University Hospital, LHSC, 339 Windermere \\ Road, London, Ontario N6A 5A5, Canada. Email: Michael.Chu@lhsc.on.ca.
}

Background: Controversy exists regarding the optimal annular stabilization technique following valve sparing aortic root reconstruction (VSRR) with no comprehensive imaging data to evaluate the natural history of aortic root graft geometry, aortic valve competency and patient prognosis post-reconstruction.

Methods: Between 2008-2017, 70 consecutive patients (mean age 56.4 16.4 years, 19.7\% females) underwent VSRR. All patients were prospectively evaluated annually with clinical follow-up, echocardiography and CT imaging. Patients were assessed for survival, freedom from reoperation, degree of regurgitation, New York Heart Association (NYHA) status and graft complications and followed up to nine years post-operatively (mean $36 \pm 21$ months).

Results: The largest increase of the aortic annulus diameter observed during the surveillance period was $2.64 \% \pm 5.4 \%$ which occurred between the second and third years of follow-up and the aortic sinuses, sinotubular junction and ascending aorta all remained relatively stable based on annual CT imaging. Echocardiographic data showed far more variability in measurements at each annual post-operative visit with far less precision compared to the CT measurements taken at the same time. Due to the large variability and greater standard deviations, no significant difference was detected between the more precise CT measurements and those from the echocardiogram images. The overall survival rate was $94.3 \%$ (66 patients) at one year. Freedom from reoperation was $98.6 \%$ (69 patients). Throughout the entire duration of followup, aortic insufficiency was identified as 0 in $46(65.7 \%), 1+$ in $19(27.1 \%), 2+$ in $4(5.7 \%), 3+$ in $0(0 \%)$ and $4+$ in $1(1.4 \%)$. Mean NYHA status was $1.1 \pm 0.3$ at most recent follow-up for all patients. CT evidence showed $97.0 \%$ (64 patients) freedom from graft complication including: endocarditis, thrombosis, embolism, aneurysm, pseudoaneurysm, dehiscence, dissection and kinking.

Conclusions: The annual imaging data presented here demonstrates stability of the Dacron aortic annuloplasty reconstruction over time, without the need for internal or external annular stabilization. CT imaging proved to be far more reliable than echocardiographic images, however given the stability, annual CT imaging is of little benefit. This is the first prospective study to compare echocardiographic, CT and clinical data following VSRR.

Keywords: Valve sparing; aortic annulus; cardiac imaging

Submitted Mar 26, 2019. Accepted for publication May 06, 2019.

doi: 10.21037/acs.2019.05.09

View this article at: http://dx.doi.org/10.21037/acs.2019.05.09 


\section{Introduction}

Although the techniques were first described nearly 30 years prior (1-5), there has been a growing level of interest in aortic valve sparing (AVS) operations and aortic valve repair over the past decade. The technical aspects to these root operations, which were previously viewed as challenging, have now been learned and broadly acquired amongst those surgical teams with interests in complex aortic root surgery $(6,7)$. Previous discussions between reimplantation and remodelling techniques have matured into more academic debates about the nuances of aortic annular stabilization (7), cusp coaptation (8), effective height (8) and optimal patient selection (9). The emerging field of aortic valve repair has adopted many concepts from AVS operations. It is well recognized that most of these patients have a dilated aortic annulus and require annular stabilization at time of AVS or aortic valve repair (7). Several techniques for aortic annular stabilization have been described including suture annuloplasty (Schaefers) (8), internal ring annuloplasty (Rankin) (9) and external ring annuloplasty by Dacron graft or proprietary expansile rings (Lansac) (7). The early and late efficacy of each of these techniques remains unknown. While late aortic insufficiency outcomes have been described, there is a paucity of longitudinal imaging data to better inform these discussions (8-11).

Both echocardiographic and computer tomography (CT) imaging can be valuable in evaluating patients that have undergone AVS to assess late geometric changes. We describe a unique series of patients undergoing AVS at a single centre whom were followed-up with serial multimodal imaging, including intra-operative photography, echocardiography and computed tomography, all performed within the same echocardiography and radiology labs.

\section{Methods}

This study was approved by the University Health Science Research Ethics Board at Western University. Between 2008 and 2017, 70 consecutive patients undergoing both emergent and elective valve sparring aortic root reconstruction were prospectively enrolled in this study. Demographic and perioperative details can be found in Tables 1 and 2, respectively. All patients were operated on by a single surgeon and evaluated annually with clinical follow-up, echocardiography and CT imaging. Patients were assessed for overall survival, event free survival, New York Heart Association (NYHA) status and degree of aortic regurgitation and over time. All echocardiograms and CT images were performed in the same labs and overread by single echocardiographer and radiologist, respectively. Patients were followed up to 9 years post-operatively (mean $36 \pm 21$ months). Annual measurements of the aortic annulus, sinuses of Valsalva, sinotubular junction and ascending aorta, from both CT and echocardiographic images, were assessed on annual follow-up. Any change observed in the diameter of these measurements is shown as a percentage of change in the diameter as compared to the previous year's measurement.

Intraoperative photographs were taken as an orthogonal view of the reconstructed aortic root prior to closing the ascending aorta. These images were evaluated by a single observer and the angles of each leaflet and commissure were measured with a caliper. A ratio was created by dividing the minimal leaflet angle by the largest leaflet angle and these values were used to track development of aortic insufficiency and stenosis over time. Patients were monitored for clinically significant outcomes defined as an aortic mean gradient $>20 \mathrm{mmHg}$ or $>2+$ aortic insufficiency, and a receiver operating characteristic (ROC) curve was created to detect any potentially predictive cut-off value of the created leaflet ratio for these outcomes.

All continuous variables were analyzed using the Wilcoxon Rank Sum test, which does not assume normality of the data set (to account for our small sample sizes). To test the hypothesis that the ratio of leaflet angles at the time of operation may adequately discriminate between patients who will develop aortic insufficiency versus those will not, we computed a ROC curve for the development of any AI versus no $\mathrm{AI}$ at two years of follow-up.

\section{Results}

\section{Early in-hospital/30-day outcomes}

AVS operation was successfully performed in all 70 patients. All operations were completed by a single surgeon using an isolated Dacron graft to downsize the aortic annulus and restore symmetry among the cusps when needed. Every case resulted in an effective cusp height $\geq 10 \mathrm{~mm}$ prior to leaving the operating room. Two patients $(2.9 \%)$ died within 30 days after surgery and no patients suffered a stroke or transient ischemic attack (TIA). One in-hospital death occurred from an ischemic bowel following mediastinitis and the other had high risk triple valve surgery and died from sepsis and overwhelming bacteremia. The remaining 


\begin{tabular}{|c|c|}
\hline Characteristics & VSRR $(n=66)(\%)$ \\
\hline Age (years), mean $\pm S D$ & $56.4 \pm 16.4$ \\
\hline Male sex & $54(81.8)$ \\
\hline $\mathrm{BMI}\left(\mathrm{cm} / \mathrm{m}^{2}\right)$, mean $\pm \mathrm{SD}$ & $27.2 \pm 4.7$ \\
\hline $\mathrm{CHF}$ & $3(4.5)$ \\
\hline MI & $2(3.0)$ \\
\hline HTN & $34(51.5)$ \\
\hline Dyslipidemia & $24(36.4)$ \\
\hline DM II & $7(10.6)$ \\
\hline Renal failure & $1(1.5)$ \\
\hline COPD & $11(16.7)$ \\
\hline CAD & $8(12.1)$ \\
\hline PVD & $6(9.1)$ \\
\hline A. Fib & $5(7.6)$ \\
\hline \multicolumn{2}{|l|}{ CVD } \\
\hline TIA & $6(9.1)$ \\
\hline Stroke & $2(3.0)$ \\
\hline \multicolumn{2}{|l|}{ CTD } \\
\hline Marfan & $7(10.6)$ \\
\hline Loeys-Dietz & $5(7.6)$ \\
\hline Other & $1(1.5)$ \\
\hline \multicolumn{2}{|l|}{ NYHA } \\
\hline 0 & 7 (10.6) \\
\hline 1 & $33(50.0)$ \\
\hline II & $15(22.7)$ \\
\hline III & $10(15.2)$ \\
\hline IV & $1(1.5)$ \\
\hline \multicolumn{2}{|l|}{ Aortic regurgitation } \\
\hline 1 & $35(57.4)$ \\
\hline II & $15(24.6)$ \\
\hline III & $10(16.4)$ \\
\hline IV & $1(1.6)$ \\
\hline Tricuspid valve & $52(78.8)$ \\
\hline Bicuspid valve & $14(21.2)$ \\
\hline
\end{tabular}

BMI, body mass index; CHF, congestive heart failure; MI, myocardial infarction; HTN, hypertension; DM II, type II diabetes mellitus; COPD, chronic obstructive pulmonary disease; CAD, coronary artery disease; PVD, peripheral vascular disease; A. Fib, atrial fibrillation; CVD, cerebrovascular disease; TIA, transient ischemic attack; CTD, connective tissue disorder; NYHA, New York Heart Association.

\begin{tabular}{ll}
\hline Table 2 Operative details & \\
\hline Variables & VSRR $(\mathrm{n}=70)(\%)$ \\
\hline Hemi-arch & $11(11.4)$ \\
\hline Total arch & $5(7.1)$ \\
\hline Elephant trunk & $15(21.4)$ \\
\hline Additional procedure & $31.4 \pm 2.8$ \\
\hline Graft size (mm), mean \pm SD & $24(34.3)$ \\
\hline Cuspal plication & $267.3 \pm 78.4$ \\
\hline CPB time (min), mean \pm SD & $216.8 \pm 58.1$ \\
\hline X-clamp time (min), mean \pm SD & $39.5 \pm 33.6$ \\
\hline DHCA time (min), mean \pm SD & $29.5 \pm 4.6$ \\
\hline HCA temp ( ${ }^{\circ}$ C), mean \pm SD & \\
\hline Urgency & $58(82.9)$ \\
\hline Elective & $7(10.0)$ \\
\hline Urgent & $4(5.7)$ \\
\hline Emergent & $1(1.4)$ \\
\hline Salvage & \\
\hline
\end{tabular}

CPB, cardiopulmonary bypass; DHCA, deep hypothermic circulatory arrest.

perioperative complications are detailed in Table 3. One patient who presented with an acute type A aortic dissection developed permanent paraplegia postoperatively. The median (interquartile range) intensive care unit and hospital lengths of stay were $1.0(1.0,2.0)$ and $6.0(5.0,10.0)$ days, respectively.

\section{Survival and follow-up outcomes}

Survival at 30 days, one year and five years was $97.1 \%$, $94.2 \%$ and $94.2 \%$, respectively (Figure 1A). Freedom from aortic complications and re-operation at 30 days, one year and five years was $100 \%, 98.2 \%$ and $96.5 \%$, respectively (Figure 1B).

\section{Perioperative outcomes}

The overall 30-day survival rate was $97.1 \%$ (68/70 patients) (Figure 1A). Freedom from reoperation was $100 \%(66 / 66$ patients). CT evidence showed $96.5 \%$ freedom from graft complication including: endocarditis, thrombosis, embolism, aneurysm, pseudoaneurysm, dehiscence, dissection and kinking at mean follow-up of 36 months 


\begin{tabular}{|c|c|}
\hline Variables & VSRR $(n=70)(\%)$ \\
\hline Death & $2(2.9)$ \\
\hline ICU LOS (days), mean \pm SD & $2.5 \pm 3.6$ \\
\hline Hospital LOS (days), mean \pm SD & $10.3 \pm 12.3$ \\
\hline Re-op bleeding & $2(2.9)$ \\
\hline A. Fib & $12(17.1)$ \\
\hline MI & $0(0.0)$ \\
\hline Arrhythmia & $1(1.4)$ \\
\hline Dialysis/renal failure & $1(1.4)$ \\
\hline Bacteremia & $1(1.4)$ \\
\hline Mediastinitis & $1(1.4)$ \\
\hline Sternal dehiscence & $1(1.4)$ \\
\hline Stroke & $0(0.0)$ \\
\hline Spinal cord injury & $2(2.9)$ \\
\hline Resp failure & $3(4.3)$ \\
\hline $\begin{array}{l}\text { A. Fib, atrial fibrillation; MI, myoc } \\
\text { stay. }\end{array}$ & tion; LOS, length $c$ \\
\hline
\end{tabular}

(Figure 1B). No patients underwent a reoperation during the follow-up period included in this study. Mean NYHA status was $1.1 \pm 0.3$ at most recent follow-up for all patients and remained stable for all years of follow-up.

\section{Aortic insufficiency and gradients}

Throughout the entire duration of follow-up, aortic insufficiency was identified as 0 in $46(65.7 \%), 1+$ in 19 patients $(27.1 \%), 2+$ in 4 patients $(5.7 \%), 3+$ in 0 patients $(0 \%)$ and $4+$ in 1 patient (1.4\%) (Figure 2A). Peak and mean aortic gradients remained stable for all patients during the follow-up period, with no development of any significant stenosis (Figure 2B). There was no significant difference between tricuspid and bicuspid aortic valves, although on average bicuspid valves had a higher mean gradient following repair (4.8 vs. $8.2 \mathrm{mmHg}$ at two years) (data not shown).

VSRR leaflet ratios were created for all patients and these values were plotted against the development of any aortic insufficiency (Figure 3A) and stenosis (Figure 3B) as measured on annual echocardiograms over time. The development of $>2+$ aortic insufficiency at two years was deemed to be clinically significant and was used as the threshold for the creation of a ROC curve, which had an area under the ROC of 0.14 (data not shown).

\section{Aortic annulus and root}

The aortic annulus appeared to be fairly stable on follow-up as observed by echocardiography during the surveillance period. The largest change noted was a diameter decrease of $7.7 \%$ during the $1^{\text {st }}$ post-operative year. However, there appeared to be significant variability in the echocardiographic measurements with a standard deviation of $15.1 \%$. The CT images showed stability of the reconstruction far more reliably, where the greatest annual change to the aortic annulus diameter of $3.3 \%$ also occurred within the $3^{\text {rd }}$ and $4^{\text {th }}$ post-operative year, with more consistency of annular measurements as demonstrated by a standard deviation of only $2.3 \%$ (Figure 4). Despite the differences noted in all of the annular measurements with these two modalities, no statistical significance was seen between the measurements of the annulus at one-, twoor four-year follow-up (P values 0.273, 0.50, and 0.317, respectively). CT imaging used to track the changes for each patient showed excellent stability from year to year with regards to annular dilation, annular area, and annular circumference (Figure 5).

Similarly, measurements taken for the sinuses of Valsalva by CT showed overall stability of the repair with the maximal annular diameter change occurring between the $3^{\text {rd }}$ and $4^{\text {th }}$ years at $2.4 \% \pm 1.0 \%$. This stability was not reflected in the echocardiographic images which measured a maximal annular change in sinus of Valsalva diameter occurring between the $2^{\text {nd }}$ and $3^{\text {rd }}$ post-operative years of $9.2 \% \pm 34.8 \%$. Again, due to the large variability and standard deviation of the echocardiographic measurements, no significant difference was detected between these two data sets at one-, two-, three- or four-year follow-up (P values 0.227 , $0.232,0.398$ and 0.18 , respectively) (Figure 4).

\section{Sinotubular junction}

With regards to the sinotubular junction, CT measurements showed annual stability with little variability. The maximal diameter change based on CT imaging occurred between the $3^{\text {rd }}$ and $4^{\text {th }}$ post-operative years and was $3.3 \% \pm 0.1 \%$. Echocardiographic imaging of the sinotubular junction also demonstrated annual stability, with slight less variability in measurements as compared to the annulus and sinuses. The maximal change in sinotubular junction diameter within one year also occurred between the $3^{\text {rd }}$ and $4^{\text {th }}$ post-operative years was $23.6 \% \pm 16.3 \%$. Again, no statistical difference 
A

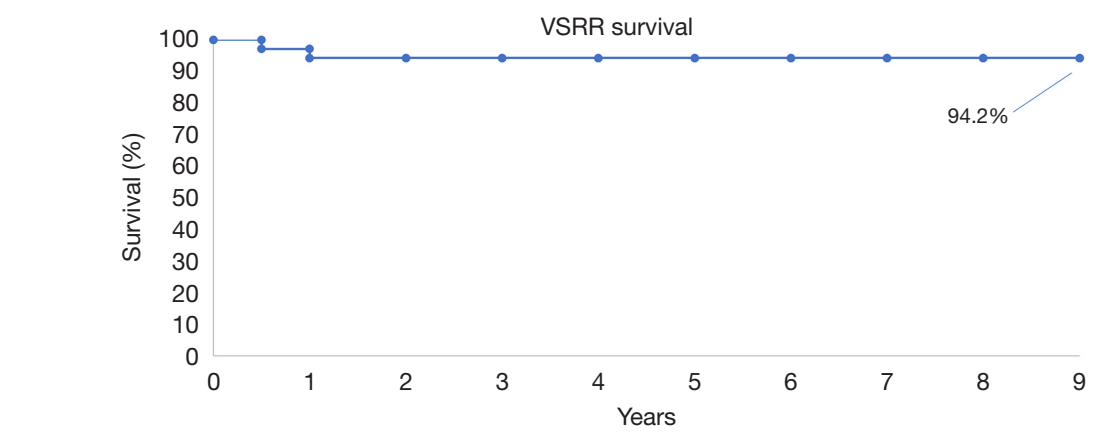

\begin{tabular}{|l|c|c|c|c|c|c|c|c|c|c|}
\hline Follow-up & Discharge & $1 \mathrm{yr}$ & $2 \mathrm{yr}$ & $3 \mathrm{yr}$ & $4 \mathrm{yr}$ & $5 \mathrm{yr}$ & $6 \mathrm{yr}$ & $7 \mathrm{yr}$ & $8 \mathrm{yr}$ & $9 \mathrm{yr}$ \\
\hline Number at risk & 68 & 62 & 50 & 39 & 21 & 8 & 3 & 2 & 1 & 1 \\
\hline Survival & $97.1 \%$ & $94.2 \%$ & $94.2 \%$ & $94.2 \%$ & $94.2 \%$ & $94.2 \%$ & $94.2 \%$ & $94.2 \%$ & $94.2 \%$ & $94.2 \%$ \\
\hline
\end{tabular}

B

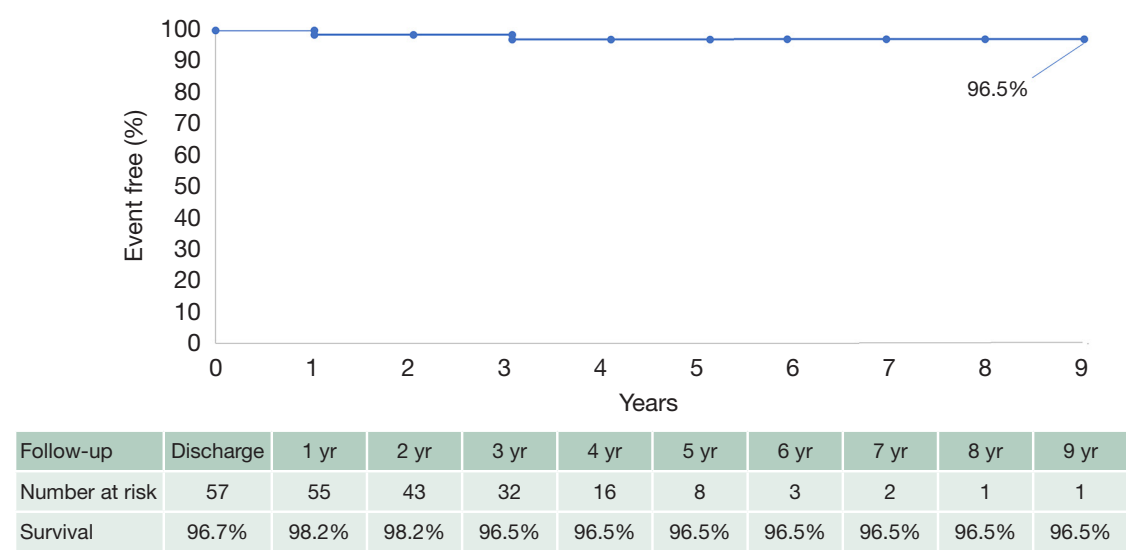

Figure 1 Kaplan-Meier survival curve of all post-operative valve sparing patients. No patients were lost to follow-up in the post-operative surveillance period (A). Kaplan-Meier curve indication any post-operative graft complications such as: endocarditis, thrombosis, embolism, aneurysm, pseudoaneurysm, dehiscence, dissection or kinking (B).

was detected between the CT and echocardiographic measurements (P values 0.593 and 0.655) (Figure 4).

CT imaging was used to monitor the STJ and showed excellent stability for the duration for the follow-up period (Figure 5).

\section{Ascending aorta}

Lastly, the ascending aorta graft proved to be stable on follow-up CT imaging. The largest diameter change within a one-year period was $1.6 \% \pm 1.5 \%$ which occurred between the $2^{\text {nd }}$ and $3^{\text {rd }}$ post-operative years. Echocardiography showed the largest diameter change of $5.6 \% \pm 7.9 \%$ during the $2^{\text {nd }}$ and $3^{\text {rd }}$ post-operative years. Again, no significant difference was shown between CT and echocardiographic measurements, likely due to greater variability (as evidenced by larger standard deviations) in the echocardiographic measurements.

\section{Leaflet ratio}

We were unable to detect any significance with regard to the different leaflet ratios and the clinically significant echocardiographic findings of any $\mathrm{AI}$ versus no $\mathrm{AI}$ at two years of follow-up. The area under our receiver operator curve was 0.28 ( $95 \%$ CI, 0.10 to 0.46 ) (data not shown).

\section{Discussion}

The results of this study again demonstrate the benefits of AVS operations with excellent operative survival (97.1\%) as well as freedom from reoperation $(98.5 \%)$ and graft 


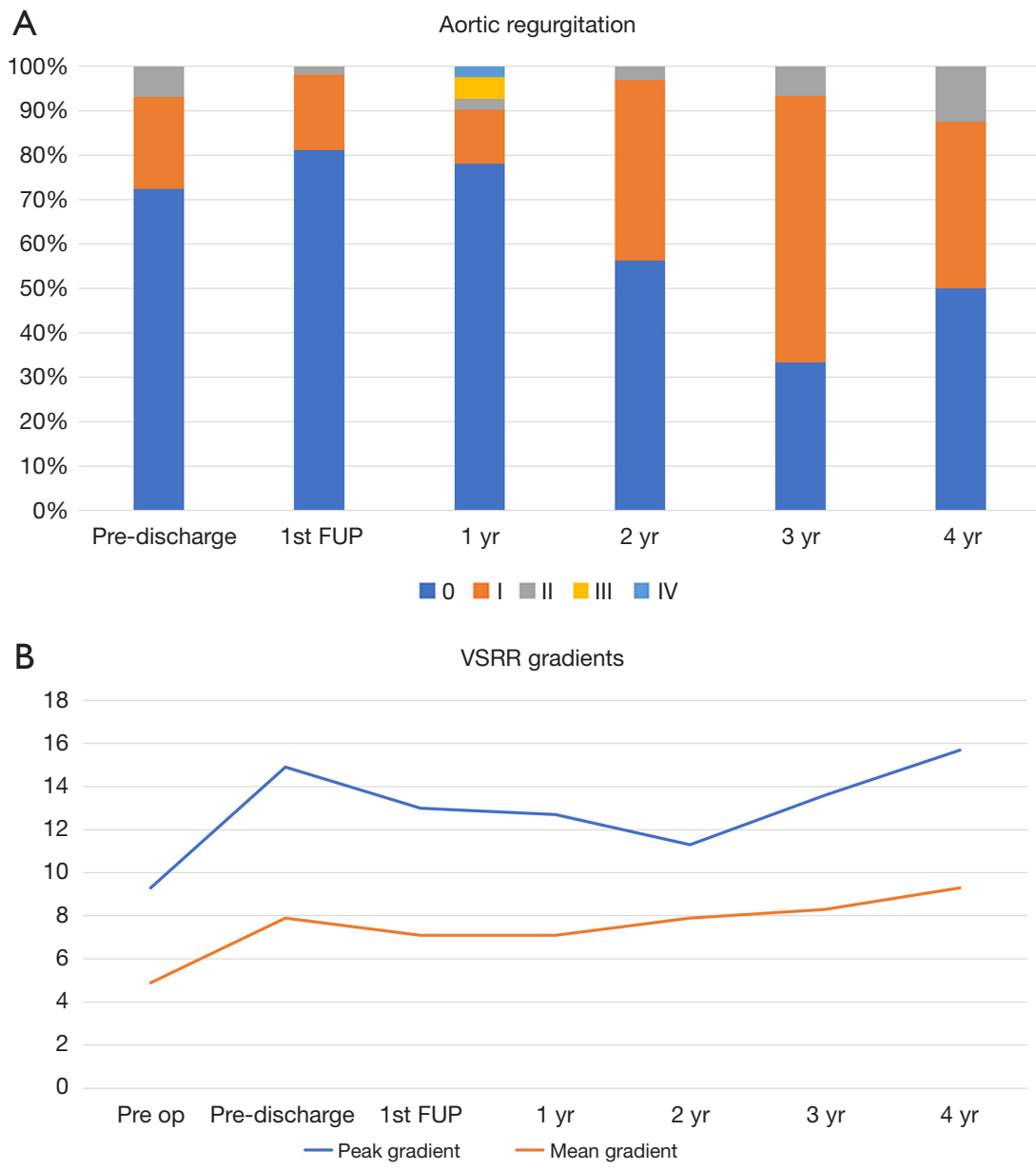

Figure 2 Degree of aortic regurgitation as represented by a percentage of the total patient population prior to discharge, at first follow-up and at each post-operative year (A). Aortic valve mean and peak gradients ( $\mathrm{mmHg}$ ), preoperatively, prior to discharge, at first follow-up and each post-operative year (B).

complications (96.5\%) (Figure 1B), which has been shown in previously published data by David and other groups (9-11). Furthermore, this study demonstrates stability of aortic valve competence throughout the duration of followup, with $92.8 \%$ of patients having either 0 or $1+\mathrm{AI}$ on annual transthoracic echocardiogram imaging along with clinical stability of patient's NYHA status with an average of 1.1 in all post-op patients.

The introduction of the leaflet ratio concept proposed here helps to quantify asymmetry among valve leaflets in both trileaflet and bileaflet aortic valves, which has typically thought to be a significant factor when determining the feasibility and durability of repair in aortic valve surgery. However, we were not able to detect a significant value with regards to a leaflet ratio that was predictive of a hemodynamically significant gradient or regurgitation following VSRR as indicated by the area under the ROC curve of 0.14 , indicating that a reliable repair is feasible even in valves that are severely asymmetrical. However, this outcome may also be a result of being under-powered due to the low incidence of adverse events and a shorter duration of follow-up. Further longitudinal data is still required to determine if a significant ratio value exists where replacement may be of a better option, but this is a quick tool that can be used to compare all valves attempting to be spared, including both bicuspid and tricuspid aortic valves.

Controversy still exists with regards to supporting the aortic annulus during aortic valve repair and the optimal techniques. Lansac and Schaefers have described the addition of an external annuloplasty ring or suture annuloplasty to help provide additional support to aortic valve repair or remodelling root replacement (12-17). 

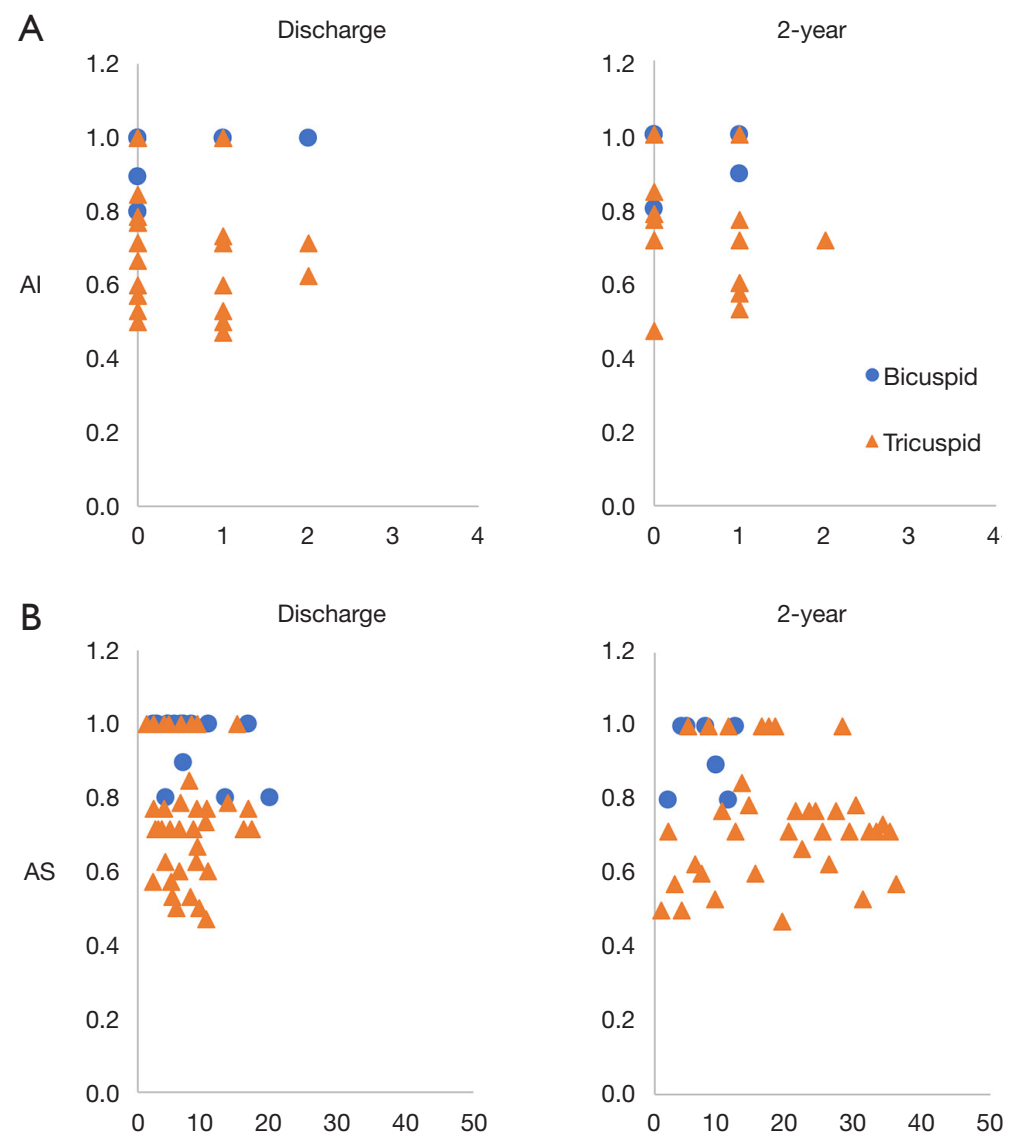

Figure 3 VSRR leaflet ratios (A) AI and (B) AS. VSRR, valve sparing aortic root reconstruction; AI, aortic insufficiency; AS, aortic stenosis.

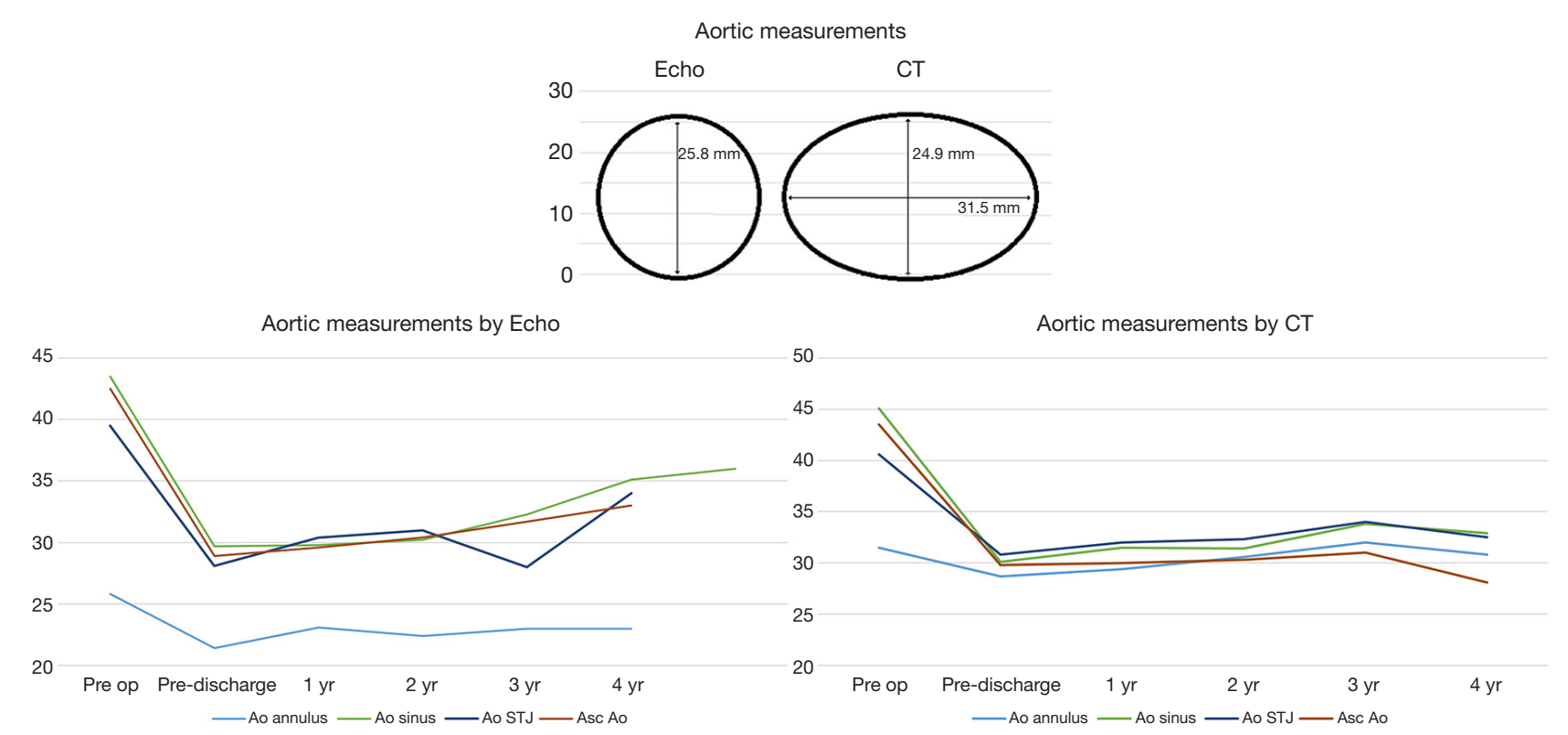

Figure 4 Aortic measurements by Echo vs. CT. 

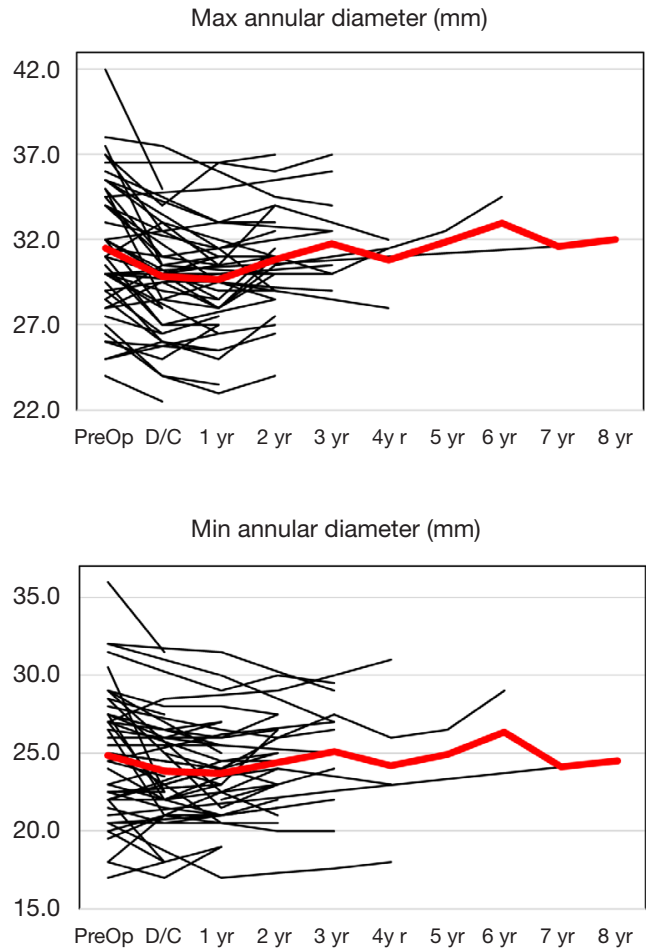

Figure 5 Annular CT measurements.

The work presented here addresses this controversy with combined complete prospective clinical and imaging follow-up data of patients undergoing a valve sparing root reimplantation to fully characterize any annular dilation and subsequent aortic insufficiency over time. The comprehensive imaging follow-up demonstrates the stability of the Dacron aortic annuloplasty, with no significant dilation of the aortic annulus or development of recurrent aortic insufficiency.

In our patient population, the aortic annulus demonstrated some small early dilatation along with an elliptical conformation; however, the sinus of Valsalva, sinotubular junction and ascending aorta generally showed little dilation of only a few percent (\%) from one year to the next. The changes observed in the diameters based on CT are likely largely due to small variations in operator observations or differences in the CT slices and likely do not represent an actual significant change in the size of the reconstructed aortic root. Conversely, echocardiographic follow-up imaging demonstrated more variability and tended to overestimate dilation of the annulus and other aortic root structures post-repair. This can be seen in the larger standard deviations of each measurement as
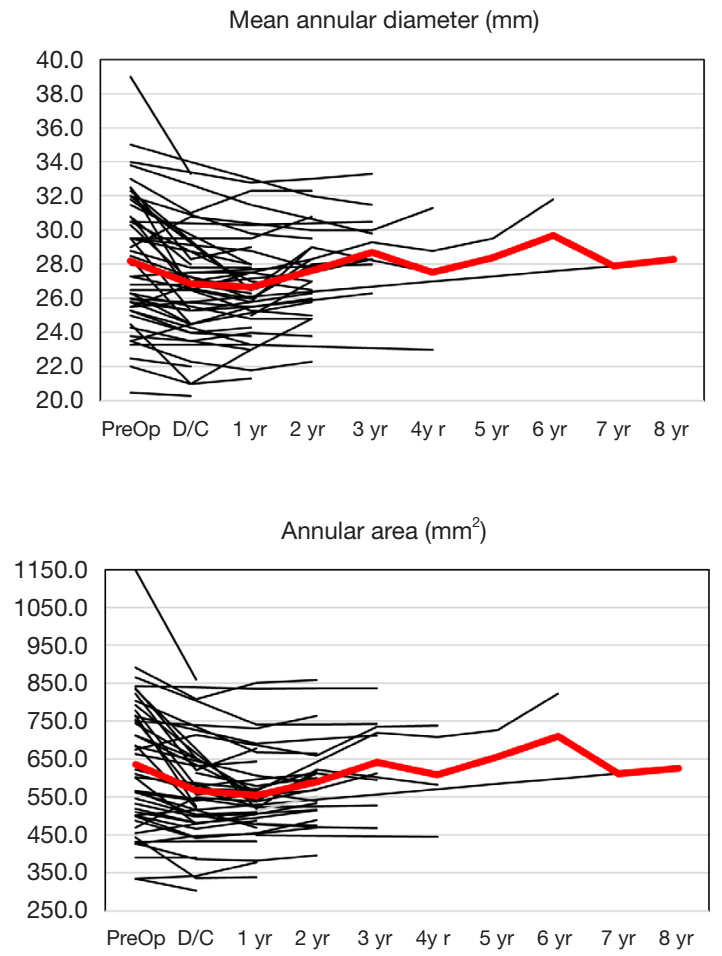

compared to the high precision measurements of the CT images. The inconsistent echo measurements are likely due to variability in suboptimal echocardiographic visualization, intra-observer technique, and non-orthogonal views. Additionally, CT evidence indicated that over time the annulus developed an elliptical configuration which may also contribute to differences seen in echocardiographic measurements, but also raises concerns that a round internal ring annuloplasty may not be well suited to provide durable stability to this elliptical configuration as compared to external ring annuloplasty or the simple Dacron graft itself, which are better suited to conform to this geometrical change. We feel this evidence supports the late durability of a Dacron ring annuloplasty to stabilize the aortic annulus.

Despite a median follow-up of 14 months, and with some patients followed as long as nine years, we have not seen any indication from this patient cohort of progressive annular/root dilation, mortality, worsening regurgitation or graft complication which has been demonstrated in other large retrospective studies (18-21). However, longer follow-up of these individuals is still needed in order to confirm the stability of the repair as the most significant decline in aortic valve competence was demonstrated 
between the $15^{\text {th }}$ and $18^{\text {th }}$ post-operative year in David's 25-year experience (19).

The results of this study are limited by the fact that there was no blinding or randomization, and small overall numbers of patients. Given the prospective descriptive design there is also no comparative group.

In conclusion, AVS is an excellent option for treating patients with aortic root aneurysms and aortic insufficiency with excellent patient survival, longevity and stability of the repair. We feel this longitudinal, comprehensive imaging study supports the durability and stability of Dacron ring annuloplasty in stabilizing the aortic annulus, albeit in an elliptical confirmation. The novel concept of the leaflet ratio was introduced here but failed to identify a significant value where the patient is at a higher risk of early valve failure. This may indicate that with contemporary valve repair techniques, even very asymmetrical valves can have a durable repair, but the low incidence of any adverse echocardiographic findings on follow-up introduces the risk of a type II error. Regardless the leaflet ratio provides surgeons with an objective indicator for describing leaflet asymmetry at the time of aortic valve surgery.

\section{Acknowledgments}

None.

\section{Footnote}

Conflicts of Interest: The authors have no conflicts of interest to declare.

\section{References}

1. David TE, Feindel CM. An aortic valve-sparing operation for patients with aortic incompetence and aneurysm of the ascending aorta. J Thorac Cardiovasc Surg 1992;103:61721; discussion 622.

2. David TE, Feindel CM, Bos J. Repair of the aortic valve in patients with aortic insufficiency and aortic root aneurysm. J Thorac Cardiovasc Surg 1995;109:345-51; discussion 351-2.

3. David TE. Remodeling the aortic root and preservation of the native aortic valve. Op Tech Cardiac Thorac Surg 1996;1:44-56.

4. Sarsam MA, Yacoub M. Remodeling of the aortic valve anulus. J Thorac Cardiovasc Surg 1993;105:435-8.

5. Yacoub MH, Gehle P, Chandrasekaran V, et al. Late results of a valve-preserving operation in patients with aneurysms of the ascending aorta and root. J Thorac Cardiovasc Surg 1998;115:1080-90.

6. Leshnower BG, Myung RJ, McPherson L, et al. Midterm results of David $V$ valve-sparing aortic root replacement in acute type A aortic dissection. Ann Thorac Surg 2015;99:795-800.

7. Lansac E, Di Centa I, Sleilaty G, et al. An aortic ring to standardise aortic valve repair: preliminary results of a prospective multicentric cohort of 144 patients. Eur J Cardiothorac Surg 2010;38:147-54.

8. Schneider U, Aicher D, Miura Y, et al. Suture Annuloplasty in Aortic Valve Repair. Ann Thorac Surg 2016;101:783-5.

9. Rankin JS, Mazzitelli D, Fischlein T, et al. Geometric Ring Annuloplasty for Aortic Valve Repair During Aortic Aneurysm Surgery: Two-Year Clinical Trial Results. Innovations (Phila) 2018;13:248-53.

10. David TE. Aortic Valve Sparing in Different Aortic Valve and Aortic Root Conditions. J Am Coll Cardiol 2016;68:654-64.

11. David, TE. Aortic valve sparing operations: outcomes at 20 years. Ann Cardiothorac Surg 2013;2:24-9.

12. Lansac E, Di Centa I, Sleilaty G, et al. Remodeling root repair with an external aortic ring annuloplasty. J Thorac Cardiovasc Surg 2017;153:1033-42.

13. Rankin JS, Gaca JG. Techniques of Aortic Valve Repair. Innovations (Phila) 2011;6:348-54.

14. Ikonomidis JS. Valve-sparing aortic root replacement: Surgeon and patient factors contribute to long-term durability. J Thorac Cardiovasc Surg 2017;153:230-1.

15. de Kerchove L, Jashari R, Boodhwani M, et al. Surgical anatomy of the aortic root: Implication for valve-sparing reimplantation and aortic valve annuloplasty. J Thorac Cardiovasc Surg 2015;149:425-33.

16. Kvitting JP, Kari FA, Fischbein MP, et al. David valvesparing aortic root replacement: Equivalent midterm outcome for different valve types with or without connective tissue disorder. J Thorac Cardiovasc Surg 2013;145:117-26, 127.e1-5; discussion 126-7.

17. David TE, Feindel CM, David CM, et al. A quarter of a century of experience with aortic valve-sparing operations. J Thorac Cardiovasc Surg 2014;148:872-9; discussion 879-80.

18. Stephens EH, Liang DH, Kvitting JP, et al. Incidence and progression of mild aortic regurgitation after Tirone David reimplantation valve-sparing aortic root replacement. J Thorac Cardiovasc Surg 2014;147:169-77, 
178.e1-178.e3.

19. David TE, Maganti M, Armstrong S. Aortic root aneurysm: principles of repair and long-term follow-up. J Thorac Cardiovasc Surg 2010;140:S14-9.

20. Lansac E, Di Centa I, Vojacek J, et al. Valve sparing root

Cite this article as: Valdis M, Thain A, Jones PM, Chan I, Chu MW. Multimodal imaging of aortic annulus and root geometry after valve sparing root reconstruction. Ann Cardiothorac Surg 2019;8(3):362-371. doi: 10.21037/acs.2019.05.09 replacement: the remodeling technique with external ring annuloplasty. Ann Cardiothorac Surg 2013;2:117-23.

21. Schäfers HJ, Aicher D. Root remodeling for aortic root dilatation. Ann Cardiothorac Surg 2013;2:113-6. 